\title{
THE
}

\section{Experimental Tropical Cyclone Prediction Using the GFDL 25-km- Resolution Global Atmospheric Model}

\author{
Jeffrey S. Gall \\ University of Rhode Island \\ Isaac Ginis \\ University of Rhode Island, iginis@uri.edu \\ Shian-Jiann Lin \\ Timothy P. Marchok \\ Jan-Huey Chen
}

Follow this and additional works at: https://digitalcommons.uri.edu/gsofacpubs

\section{Citation/Publisher Attribution}

Gall, J. S., Ginis, I., Lin, S.-J., Marchok, T. P., \& Chen, J.-H. (2011). Experimental Tropical Cyclone Prediction Using the GFDL 25-km-Resolution Global Atmospheric Model. Weather and Forecasting, 26 1008-1019. doi: 10.1175/WAF-D-10-05015.1

Available at: http://dx.doi.org/10.1175/WAF-D-10-05015.1

This Article is brought to you for free and open access by the Graduate School of Oceanography at DigitalCommons@URI. It has been accepted for inclusion in Graduate School of Oceanography Faculty Publications by an authorized administrator of DigitalCommons@URI. For more information, please contact digitalcommons-group@uri.edu. 


\title{
Experimental Tropical Cyclone Prediction Using the GFDL 25-km-Resolution Global Atmospheric Model
}

\author{
JEFFREY S. GALL* AND ISAAC GINIS \\ University of Rhode Island, Narragansett, Rhode Island \\ ShiAn-JiAnn Lin, Timothy P. MARChoK, AND JAN-Huey Chen \\ Geophysical Fluid Dynamics Laboratory, Princeton, New Jersey
}

(Manuscript received 15 August 2010, in final form 4 June 2011)

\begin{abstract}
This paper describes a forecasting configuration of the Geophysical Fluid Dynamics Laboratory (GFDL) High-resolution Atmospheric Model (HiRAM). HiRAM represents an early attempt in unifying, within a global modeling framework, the capabilities of GFDL's low-resolution climate models for Intergovernmental Panel on Climate Change (IPCC) type climate change assessments and high-resolution limited-area models for hurricane predictions. In this study, the potential of HiRAM as a forecasting tool is investigated by applying the model to the near-term and intraseasonal hindcasting of tropical cyclones (TCs) in the Atlantic basin from 2006 to 2009. Results demonstrate that HiRAM provides skillful near-term forecasts of TC track and intensity relative to their respective benchmarks from $t=48 \mathrm{~h}$ through $t=144 \mathrm{~h}$. At the intraseasonal time scale, a simple HiRAM ensemble provides skillful forecasts of 21-day Atlantic basin TC activity at a 2-day lead time. It should be noted that the methodology used to produce these hindcasts is applicable in a real-time forecasting scenario. While the initial experimental results appear promising, the HiRAM forecasting system requires various improvements in order to be useful in an operational setting. These modifications are currently under development and include a data assimilation system for forecast initialization, increased horizontal resolution to better resolve the vortex structure, 3D ocean model coupling, and wave model coupling. An overview of these ongoing developments is provided, and the specifics of each will be described in subsequent papers.
\end{abstract}

\section{Introduction}

Global forecasting models and their data assimilation systems, even at much lower resolution, are critical to weather prediction at the regional scale. This is particularly true at longer lead times when forecasts are strongly influenced by information entering-leaving the artificially imposed lateral boundaries in the limited-area models. Forecasts from global models provide, at the very least, the lateral boundary conditions for high-resolution, limitedarea forecast models. Continued advancements in numerical algorithms and computing power allow global

\footnotetext{
* Additional affiliation: Geophysical Fluid Dynamics Laboratory,
} Princeton, New Jersey.

Corresponding author address: Jeffrey S. Gall, Geophysical Fluid Dynamics Laboratory, 201 Forrestal Rd., Princeton, NJ 08540.

E-mail: jeffrey.gall@noaa.gov models to be run at ever-increasing horizontal and vertical resolutions, and for longer model forecast integrations. The National Weather Service's (NWS) global spectral model (the Global Forecast System, GFS; Lord 1993), for example, has increased its horizontal resolution from $180 \mathrm{~km}$ in 1980 to $35 \mathrm{~km}$ as of year-end 2009 (information online at http://www.emc.ncep.noaa.gov/ $\mathrm{gmb} / \mathrm{STATS} / \mathrm{html} /$ model_changes.html). It is likely that within a decade, global forecasting models will be run operationally at the same resolution as the current stateof-the-art regional forecasting models (i.e., sub-10-km horizontal resolution). Therefore, the weather forecasting community should develop and systematically evaluate the predictability of higher-resolution global models both for mesoscale weather events and for longer forecast lead times.

Forecasting tropical cyclones (TCs) at the intraseasonal time scale (7-28 days) represents an excellent test bed for evaluating a high-resolution global model. It is of particular interest to the climate modeling community, 
and even the general public, that a global model that has been developed, tuned, and used for climate change studies also be demonstrated to possess skill at forecasting severe weather events in an operational-like setting.

This paper introduces an early version of the HighResolution Atmosphere Model (HiRAM) developed at the National Atmospheric and Oceanic Administration/ Geophysical Fluid Dynamics Laboratory (NOAA/GFDL) and applied to forecasting TC activity in the Atlantic basin. As described in more detail in section 2a, HiRAM is uniquely formulated when compared to the current generation of operational models. The finite-volume dynamical core is based on the nonhydrostatic extension of the vertically Lagrangian discretization (Lin 2004) with the horizontal discretization reformulated on the cubed-sphere grid (Putman and Lin 2007) for enhanced scalability on modern parallel computers (e.g., SGI, Cray, and IBM platforms). We have striven to construct the physical parameterizations in a more "resolution flexible" way such that only a few changes to the inputs are required for it to be run at vastly different horizontal resolutions ranging from "cloud resolving" $(1-5 \mathrm{~km})$ to a more typical "high resolution" climate model (100 km). This contrasts with operational global numerical weather prediction (NWP) or climate models, which require significant modifications to many of their physical parameterizations any time the horizontal resolution is upgraded.

A plethora of dynamical models are available as forecast guidance on TC track in the Atlantic basin. Current operational global forecasting models include the Met Office Global Model (UKM; Cullen 1993; Heming et al. 1995), the NCEP GFS model, the Naval Operational Global Atmospheric Prediction System (NOGAPS; Hogan and Rosmond 1991; Goerss and Jeffries 1994), the European Centre for Medium-Range Weather Forecasts (ECMWF) model (e.g., Vitart 2004), and the Canadian Meteorological Centre Global Environmental Multiscale Model (CMC; e.g., Côté et al. 1998). All of these global models utilize sophisticated data assimilation systems and feature skillful TC track forecasts for the Atlantic basin relative to a climatology-persistence model forecast of TC track (CLP5; Aberson 1998) out to 5 days (Franklin 2010). Limited-area models for TCs have sufficiently large domains that encompass the area of influence of a TC for a period of a few days and obtain their boundary conditions from a global model. Two such operational models used in forecasting TC track for the Atlantic basin are the National Weather Service (NWS) GFDL Hurricane Model (e.g., Bender et al. 2007) and the Hurricane Weather Research and Forecast (HWRF) model developed by the NCEP/Environmental Modeling Center (e.g., Davis et al. 2008). Like the global models, these limited-area operational models produce skillful TC track forecasts for the Atlantic basin out to 5 days (Franklin 2010).

As noted in DeMaria et al. (2007), the errors of the official TC track forecasts in the Atlantic basin are an order of magnitude smaller than the errors of the official TC intensity forecasts. Such a result indicates that intensity forecasting still has more room for improvement. Many of the global operational models used for TC track forecasting in the Atlantic are handicapped by the resolution, initialization, and parameterizations of the smaller-scale processes (Knaff et al. 2007). As a result, these models cannot adequately resolve the inner core of a TC, which ultimately leads to little skill in forecasting TC intensity (DeMaria et al. 2007). The baseline for TC intensity forecasts in the Atlantic basin is the 5-day Statistical Hurricane Intensity Forecast model (SHF5; Knaff et al. 2003), the climatology and persistence model for intensity. As seen in Franklin (2010), the maximum skill of dynamical model TC intensity forecasts relative to SHF5 is between $20 \%$ and $40 \%$ for up to 5 days, which is about 2 times smaller than the maximum skill of dynamical models for TC track forecasts relative to CLP5. It should be noted that as of 2009, the regional GFDL hurricane model has been the only dynamical model that consistently provides both skillful intensity and track forecasts.

In recent years significant efforts have focused on seasonal predictions of TC activity using dynamical global models (see Camargo 2007 for a review of these). Surprisingly few resources have been spent examining the predictability of TC activity on the intraseasonal time scale (defined here as the period between 7 and 28 days). Of the schemes used to predict TC activity at lead times greater than 1 week, most utilize a statistical methodology. Roundy (2008) employed a statistical model based on both filtering in the wavenumber-frequency domain and extended empirical orthogonal functions (Roundy and Schreck 2009) to make probabilistic forecasts that a TC of some intensity would be located within $5^{\circ}$ of a given latitude and longitude point at lead times out to 2 weeks (information online at http://www.atmos. albany.edu/facstaff/roundy/tcforecast/tcforecast.html). LeRoy and Wheeler (2008) developed a logistic regression technique to predict the probability of TC formation in the South Pacific Ocean Basin. The model predictors included the climatological seasonal cycle of TC activity, the phase of the Madden-Julian oscillation (MJO; e.g., Zhang 2005), and SST. Their forecasts are shown to be skillful relative to climatology out to 4 weeks. The tropical group at Colorado State University (CSU) began issuing intraseasonal forecasts of Atlantic basin TC activity starting with the 2009 season (information online at http://hurricane.atmos.colostate.edu/ 


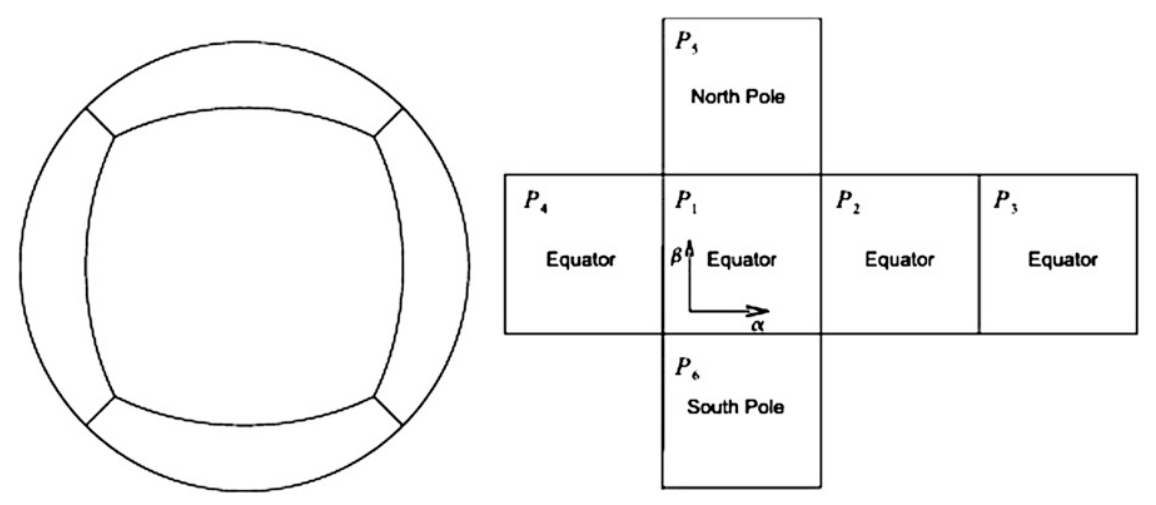

FIG. 1. Schematic illustrating the cubed-sphere grid.

Forecasts/2009/nov2009/nov2009.pdf). The CSU tropical group provides a biweekly forecast of the accumulated cyclone energy (ACE) index for the proceeding 14 days. Their 2-week ACE forecast for the Atlantic basin is composed of the following predictors: global model forecasts, the current and projected states of the MJO, and their current seasonal forecast of basin-wide ACE. The forecasts also account for current active TCs in the Atlantic basin and the National Hurricane Center (NHC) tropical weather outlook. Klotzbach (2010) demonstrated that the performance of these intraseasonal forecasts is largely dependent on the forecast skill of the MJO.

The utility of dynamical models as an intraseasonal forecasting tool of TC activity has just recently begun to be investigated within the tropical research community. Belanger et al. (2010) examined the predictability of intraseasonal TC activity with the monthly ECMWF 51-member coupled ensemble. Results indicate that the modeling system can broadly capture large-scale regions of higher-lower probability of TC activity. In particular, the ensemble system demonstrated forecast skill relative to climatology on the intraseasonal time scale for the Caribbean Sea and the Main Development Region of the Atlantic. Intraseasonal TC activity in the Atlantic was shown to vary with the phase and intensity of the MJO. Elsberry et al. (2010) also made use of the 51-member ECMWF ensemble to examine the predictability of TC activity between 10 and 30 days. It was concluded that the ECMWF ensemble provides guidance on the formation and track of TCs at the intraseasonal time scale, at least for the strongest TCs occurring during the peak of the hurricane season. The ensemble system was less skillful for early and late season TCs as well as storms that never reached a significant intensity. The findings of Vitart et al. (2010) support the notion of intraseasonal dynamical forecasts of TC activity, as the authors' dynamical forecasts of 1-week TC activity for the South Pacific were shown to be skillful relative to an intraseasonal statistical model counterpart.

Section 2 of this paper provides a brief description of HiRAM including both the TC-breeding procedure and method for initialization. Section 3 presents near-term HiRAM hindcasts of both Atlantic basin TC track and intensity out to 5 days for portions of the 2006-09 seasons. Section 4 highlights HiRAM hindcasts of Atlantic basin intraseasonal TC activity out to 28 days. Section 5 summarizes the performance of the HiRAM hindcasts and provides a discussion of ongoing model and data assimilation improvements/developments, which should ultimately lead to improved forecast skill.

\section{Methodology}

\section{a. Model description}

A forecasting configuration of HiRAM developed at GFDL (e.g., Zhao et al. 2009) is applied to Atlantic basin TC activity at both the near-term (up to 5 days) and intraseasonal time scales (from 7 to 28 days). HiRAM features a finite-volume dynamical core formulated on a cubed-sphere grid (Putman and Lin 2007). A schematic of this grid topology is presented in Fig. 1. The cubed-sphere grid is a projection of a cube onto the surface of a sphere represented as six adjoining equal-sized grid faces. Four faces of the cube align over the equator, and the remaining two faces cover the north and south polar regions. The cubed-sphere grid results in excellent grid uniformity, and eliminates numerical issues with strong grid singularity at the poles. This new dynamical core provides a modest increase in overall numerical accuracy while at the same time improving the scalability of the model when using large numbers of processors (e.g., Putman and Lin 2007).

As was done in Zhao et al. (2009, hereafter Z09), a number of important changes have been made to version 
(a)

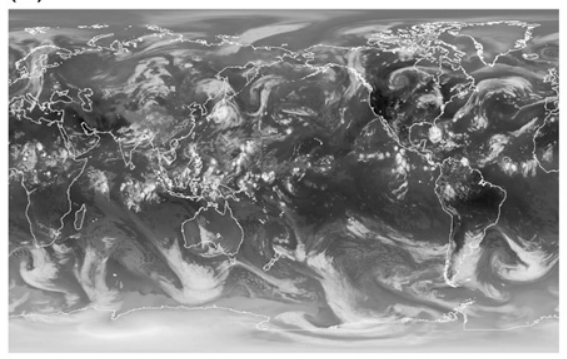

(c)

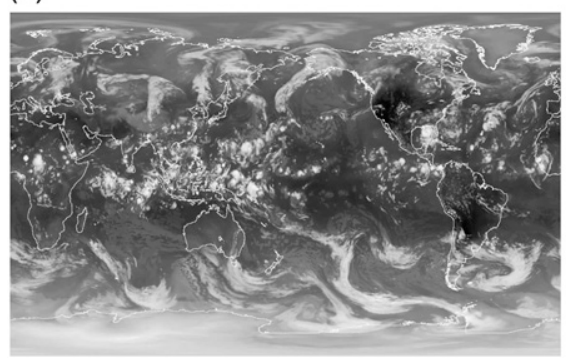

(b)

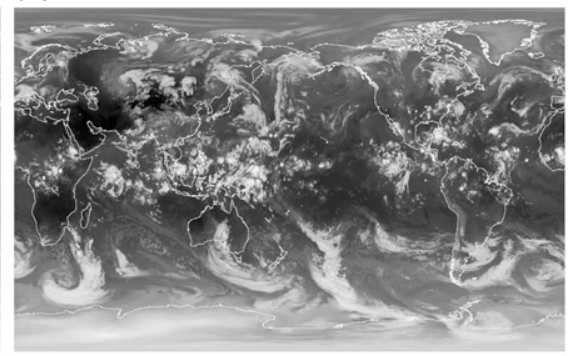

(d)

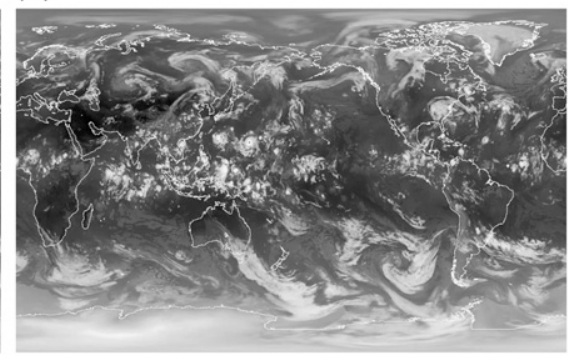

FIG. 2. Snapshots of the model-derived OLR at $t=(\mathrm{a})+1,(\mathrm{~b})+3,(\mathrm{c})+5$, and (d) +7 days. The model hindcast was started at 0000 UTC 24 Aug 2005, and times are provided relative to the model start time.

2.1 of the GFDL's Atmospheric Model (AM; Anderson et al. 2004) for these, and future, high-resolution weather and climate applications. These changes include the following components.

\section{- A further increase in horizontal resolution}

The $50-\mathrm{km}$ resolution utilized by Z09 is adequate for establishing an excellent TC climatology with realistic seasonal and interannual cycles. For shortterm track and intensity forecasts, it is found that a 25 $\mathrm{km}$ grid spacing provides a resolution capable of producing skillful TC intensity forecasts. Such a result agrees with the findings of Shen et al. (2006) and Lin et al. (2006) in which mesoscale-resolving GCMs run at comparable horizontal resolutions $(10-50 \mathrm{~km})$ were utilized to produce skillful forecasts of Hurricane Katrina. For this study, we are limited to a resolution of $25 \mathrm{~km}$ owing to the amount of available computer resources. Figures $2 \mathrm{a}-\mathrm{d}$ illustrates the finescale detail capable with a horizontal resolution of $25 \mathrm{~km}$.

- A new microphysics scheme suitable for a wide range of horizontal resolutions

In Z09, the probability density function (PDF) based cloud microphysics follows the standard GFDL atmospheric model (AM2.1) and contains only three water substances (specific humidity, cloud water, and cloud ice). For general applicability, particularly at or near the cloud-resolving scale, a more advanced sixclass bulk cloud microphysics (e.g., Lin et al. 1983) is incorporated. In our implementation of the Lin et al. (1983) scheme, the terminal fall of rain, graupel, snow, and cloud ice are treated with a high-order, conservative, Lagrangian remapping scheme, which leads to significant improvements in computational efficiency. This scheme is numerically identical to the vertical Lagrangian remapping method introduced by Lin (2004) and used in the aforementioned finitevolume dynamical core. Further, diffusive errors are reduced when compared to the typical highly diffusive, first-order, upwind-differencing schemes used in most regional cloud-resolving models. As in Z09, a simple PDF approach is also adopted to allow subgrid cloud formation when the mean grid relative humidity is beyond a resolution-dependent threshold. This initiation threshold is set to $90 \%$ for horizontal resolutions of $25 \mathrm{~km}$.

As described in Miura et al. (2007), the major difficulty in simulating the MJO with a global climate model is developing a cumulus parameterization that properly distributes heat and moisture by unresolved convective clouds. Proper representation of the MJO in global models is crucial because the MJO is a major source of TC predictability on the intraseasonal time scale (e.g., LeRoy and Wheeler 2008; Belanger et al. 2010). Miura et al. (2007) argue, however, that global cloud-resolving models with specified SSTs (similar to the configuration used in HiRAM) allow for realistic MJO simulations because convective activity 
can be directly linked to dynamic and thermodynamic atmospheric conditions of large-scale atmospheric circulation and convection. Given HiRAM's sophisticated microphysics scheme, it is believed that HiRAM is capable of long-range MJO forecasting and, ultimately, intraseasonal TC activity.

- An initialization scheme via large-scale nudging

As previously noted, an independent data assimilation system for the initialization of the forecast system does not yet exist. Rather, the model is initialized by nudging the principal dynamical fields (zonal wind, meridional wind, and temperature) toward the NCEP analysis up until the model forecast start time. The $2 \Delta x$ waves are removed from the tendencies arising from the nudging procedure in order to ensure that the large-scale features at the initial forecast time are highly correlated to the NCEP analysis. This procedure also provides physically consistent specific humidity and microphysical fields not available in the NCEP analysis. That is, certain fields that are crucial to the proper performance of the microphysical parameterization (e.g., cloud water and cloud ice) are "spun up" during the nudging period. The nudging time scale ( $e$-folding time) used in this study is $6 \mathrm{~h}$.

- $A$ vortex breeding scheme

As of this writing, the resolution of the NCEP analysis cannot sufficiently resolve the TC scale. As a result, TC intensity is often underestimated in the analysis. To overcome this deficiency, a 4D (3D in space and 1D in time) vortex breeding scheme is initiated 1 day prior to the initial forecast time. The latitude-longitude position and minimum sea level pressure (SLP) for all operationally tracked TCs occurring during the 1-day breeding period are obtained from the NHC and/or NCEP ftp page. ${ }^{1}$ The SLP data serve as the "observations" to which the model is nudged in time and space. The radial SLP distribution is assumed to be Gaussian, with the width interactively determined by the simulated vortex in the model. To allow some built-in uncertainty in the SLP "observations," two approximately Gaussian profiles of SLP bounding the observed SLP profile are created. The model's surface pressure is only nudged when the radial distribution of the SLP field of the vortex lies outside to bounding SLP profiles. This scheme is termed vortex breeding because the gradual "spinup" of the vortex over the 1-day breeding period is consistent with the model dynamics and physical parameterizations. Given ample nudging time

\footnotetext{
${ }^{1}$ These data are available in real time.
}

(12 $\mathrm{h}$ or more; thus, the 24-h breeding time is considered adequate), the model will generate a vortex that is close to the observed intensity. It should be noted that this procedure works best when the model resolution is capable of supporting the observed intensity. That is, under ideal conditions and given a long enough integration time, the model could spin up a TC with an intensity matching that of the observations without the use of the vortex breeding scheme.

- SST anomaly persistence scheme

Since HiRAM is used for intraseasonal forecasts, maintaining a constant SST over the course of the simulation is not a viable option, and an SST anomaly persistence scheme is utilized instead. At the initial forecast time, the SST anomaly $\left(\operatorname{SST}_{a}\right)$ at $t=0$ is calculated using

$$
\mathrm{SST}_{a}=\mathrm{SST}_{\text {analysis }}-\mathrm{SST}_{\text {climo }}
$$

where $\mathrm{SST}_{\text {anlaysis }}$ is the SST from the NCEP analysis at the initial forecast time and $\mathrm{SST}_{\text {climo }}$ is the climatological SST at the initial forecast time. Over the course of the model integration, $\mathrm{SST}_{a}$ is kept constant, and the SST at any forecast time is calculated by adding $\mathrm{SST}_{a}$ to the climatological-varying SST. That is,

$$
\operatorname{SST}(t)=\operatorname{SST}_{\text {climo }}(t)+\operatorname{SST}_{a} .
$$

During the forecast period, the SST variation is completely described by the variation in the climatological SST.

\section{b. TC tracker}

Since this study quantifies TC activity out to the intraseasonal time scale, a robust and objective TC tracking algorithm is required to ensure proper identification of all modeled TCs. The tracker used in this study is similar to the one that has been used operationally at NCEP since 1998 (Marchok 2002). It produces position fixes for several low-level parameters: relative vorticity at 850 and $700 \mathrm{mb}$, mean sea level pressure, geopotential height at 850 and $700 \mathrm{mb}$, and the minimum in the wind speed at 850 and $700 \mathrm{mb}$. To locate a maximum or minimum value for a given variable, the tracker employs a single-pass Barnes analysis (Barnes 1964) at grid points in an array centered initially on the NHC-observed position of the storm. The Barnes analysis provides an array of Gaussian weighted-average data values surrounding the initial-guess position. The position fix is defined as the point at which the Barnes function is maximized or 
minimized, depending on the parameter being analyzed. After a position fix is returned from the first iteration of the Barnes analysis, additional iterations are performed, each time centering the Barnes analysis grid on the position fix from the previous iteration, and each time halving the grid spacing of the Barnes analysis grid in order to obtain a position fix on as fine resolution a grid as possible. The position fixes for all parameters are then averaged together in order to produce a mean position fix for each forecast hour. Parameters with position fixes that are not within a specified distance (usually $275 \mathrm{~km}$ ) from the guess position for a given forecast hour are not included in the computation of the mean position fix.

Once a cyclone has been detected and is being tracked throughout the forecast, an algorithm is applied at each lead time in order to assess the thermodynamic phase of the cyclone. For this purpose, a simple procedure is used to determine the existence and strength of a mid- to upper-level warm anomaly near the storm. First, the same Barnes tracking scheme described above is applied to the 300-500-mb mean temperature field within $275 \mathrm{~km}$ of the storm center in order to locate the center of the warm anomaly, similar to Vitart et al. (1997). Once a center location has been found, a check is made to determine whether or not a closed contour in that mean temperature field surrounds the warm anomaly. This check is done to ensure that a minimum level of organization is present in the warm anomaly. We found that using a contour interval of $1 \mathrm{~K}$ produced consistent and reasonable results for the HiRAM simulations.

\section{c. Experimental design}

All simulations are performed at Oak Ridge National Laboratory on their high-performance computing system (Jaguar). At the time of this writing, Jaguar is the most powerful supercomputer in the world for open scientific use and features over 125000 processing cores, $62 \mathrm{~TB}$ of memory, $600 \mathrm{~TB}$ of disk space, and a peak performance of 263 teraflops $\mathrm{s}^{-1}$. Once-daily, 0000 UTC HiRAM hindcasts are performed from 26 July through 16 September for the years 2006-09. The 48 simulations per season results in 192 total hindcasts. This portion of each season is selected because it covers the climatological maximum in TC activity for the Atlantic Ocean basin. Each simulation features a 1-day nudging period prior to the initial forecast time. As described previously, over this 1-day spinup period, all available TC data are ingested into the vortex breeding scheme to enhance the vortex scale, and the model $u, v$, and $T$ fields are nudged toward the corresponding large-scale analysis fields. HiRAM is then integrated forward in time, free of influence of any future data (even the model's ozone and $\mathrm{CO}_{2}$ datasets are based on climatology), for a period of 28 days. The land model does not have any nudging or assimilation and is driven passively (during the atmospheric nudging cycle) by the "nudged" atmospheric state. It should be noted that this procedure can easily be replicated for real-time near-term and intraseasonal forecasts of Atlantic basin TC activity.

For the near-term HiRAM hindcasts, only model forecast data out to 5 days are analyzed. As described in the previous section, the TC tracker is used to generate TC track and intensity forecasts for each operationally identified TC. For the intraseasonal HiRAM hindcasts, the research version of the TC tracker was used to identify all TC-like disturbances regardless of their operational designation over the entire 28-day model integration period.

\section{5-day forecast results}

HiRAM TC track and intensity forecasts in the Atlantic Ocean basin are evaluated against a basket of other dynamical models including the GFS and NOGAPS global models, as well as the GFDL limited-area hurricane model. Track forecasts are benchmarked against CLP5, the 5-day climatology and persistence model for TC track in the Atlantic. Figure 3 shows the best track of all TCs in the 2006-09 period used in HiRAM verification. As seen in Fig. 3, the myriad of tracks occur within many distinct regions of the Atlantic and represent a diverse sampling of short- and long-lived TCs as well.

Figure 4 gives the numbers of cases that were used in the HiRAM track and intensity verification at forecast lead times of $0,+12,+24,+36,+48,+72,+96$, and $+120 \mathrm{~h}$. As expected, the largest number of cases occurs for the initial forecast (122), and decreases monotonically to 33 cases at a forecast lead time of $+120 \mathrm{~h}$.

To determine the statistical significance of the model difference at the various forecast lead times for both track and intensity, a two-sample $t$ test is utilized. Following a procedure outlined in Wilks (2006), adjustments are made to the test statistic to account for time dependence between model forecasts (i.e., persistence) for the same TC. When calculating the test statistic, the number of samples $n$ is adjusted to reflect the equivalent number of independent samples $n^{\prime}$ (Fig. 4). Even at $120 \mathrm{~h}$, the equivalent number of independent samples $(\sim 28)$ is sufficiently large to draw meaningful conclusions on HiRAM's performance at this lead time.

\section{a. Track}

Figure 5a shows the absolute forecast track error for HiRAM along with the track error for the GFS, NOGAPS, and GFDL models. Despite the vortex 


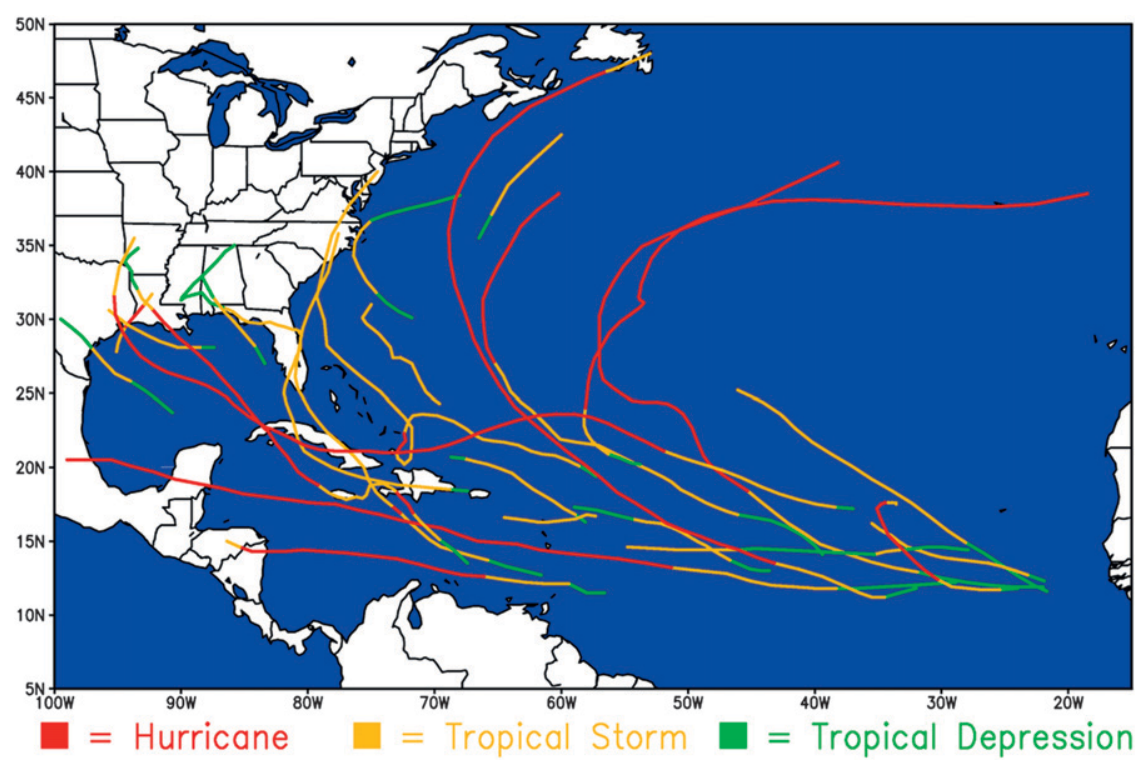

FIG. 3. Observed tracks of certain named storms in the Atlantic Ocean basin from 2006 to 2009. Only TCs for which HiRAM was verified against are plotted. Twenty-five named storms were utilized in the near-term TC forecast verification.

breeding scheme, the large initial error for HiRAM $(35 \mathrm{~nm})$ is significantly larger than the remainder of the model guidance. NOGAPS has the next largest initial track error with a value of 20 nautical miles ( $\mathrm{n}$ mi; where $1 \mathrm{n} \mathrm{mi}=1852 \mathrm{~m})$, which is $15 \mathrm{n}$ mi smaller than the initial HiRAM error. Between $t=0$ and $t=48 \mathrm{~h}$, HiRAM underperforms the dynamical model guidance, with track errors at least $25 \mathrm{n}$ mi larger than the next

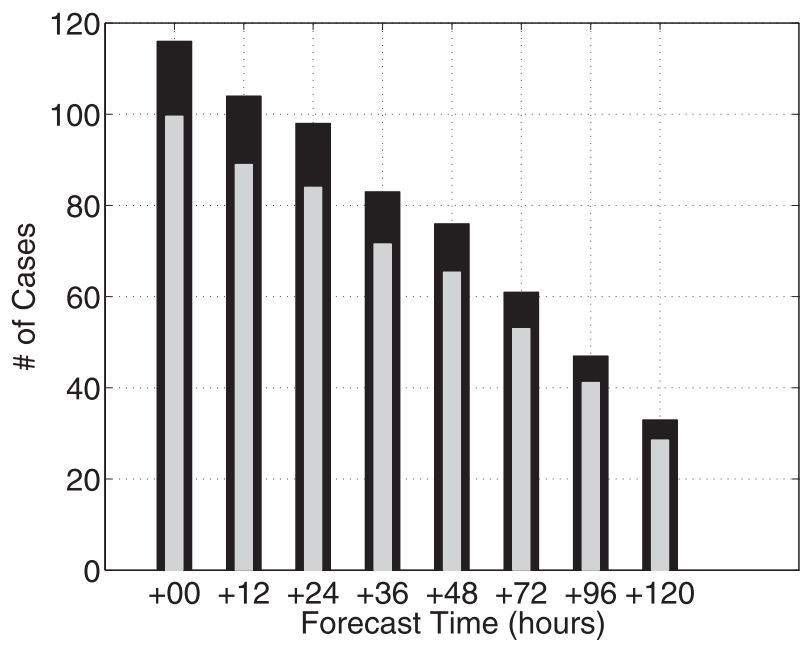

FIG. 4. Number of cases ( $n$, exterior bars) for each forecast time used in the HiRAM near-term forecast verification. Also plotted is the number of equivalent independent samples ( $n^{\prime}$, interior bars) used in assessing the statistical significance of the model difference. The number of cases is the same at all forecast times for both the forecast track and intensity verification. largest model track error. From $t=24 \mathrm{~h}$ to $t=48 \mathrm{~h}$, HiRAM forecast skill relative to CLP5 ranges from $15 \%$ to $41 \%$ (Fig. 5b). Over this time period, the performance of the selected dynamical model guidance relative to CLP5 is significantly higher $(40 \%-60 \%)$. HiRAM's underperformance at these early forecast times is most likely due to the use of the vortex initialization procedure without the sufficient horizontal resolution necessary to support the observed intensity and spatial structure.

From $t=72 \mathrm{~h}$ through $t=120 \mathrm{~h}$, the HiRAM forecast track error improves relative to both the NOGAPS
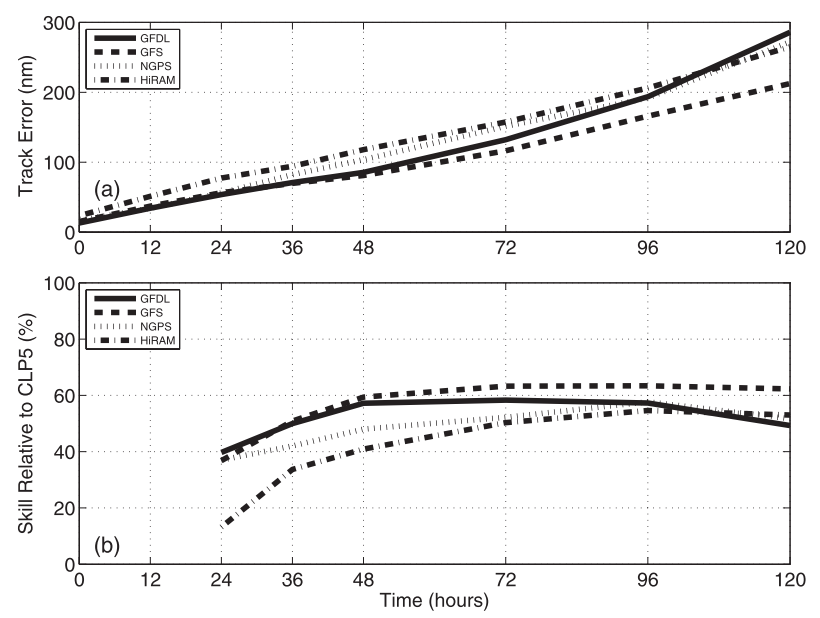

FIG. 5. (a) TC forecast track error (n mi) and (b) TC forecast track skill relative to CLP5 (\%) for HiRAM, GFDL, GFS, and NOGAPS from 2006 to 2009. 
TABLE 1. Probability of model forecast track difference between HiRAM and each of the following models: GFDL, GFS, NOGAPS, and CLP5 at various forecast lead times.

\begin{tabular}{ccccc}
\hline \hline Forecast time $(\mathrm{h})$ & GFDL & GFS & NOGAPS & CLP5 \\
\hline+24 & 1.00 & 1.00 & 1.00 & 0.90 \\
+36 & 1.00 & 1.00 & 0.97 & 1.00 \\
+48 & 1.00 & 1.00 & 0.95 & 1.00 \\
+72 & 0.98 & 1.00 & 0.72 & 1.00 \\
+96 & 0.69 & 0.96 & 0.81 & 1.00 \\
+120 & 0.68 & 0.91 & 0.57 & 1.00 \\
\hline
\end{tabular}

and GFDL forecast track errors. This decrease in track error, however, is only statistically significant against the GFDL model at $t=72 \mathrm{~h}$ (Table 1). HiRAM's superior modeling of the large-scale environmental flow, and the simultaneous initial condition error growth saturation, are the most likely reasons for the observed track forecast improvement relative to both NOGAPS and GFDL at these later lead times By $t=120 \mathrm{~h}$, the HiRAM forecast track error is $260 \mathrm{n} \mathrm{mi}$, which is 15 and $25 \mathrm{n} \mathrm{mi}$ smaller, respectively, than for the GFDL and NOGAPS forecast track errors. Additionally, HiRAM has 50\% skill relative to CLP5 by $t=120 \mathrm{~h}$, which is statistically significant at the 99th percentile. The HiRAM forecast track skill relative to CLP5 at this time is between 5\% and $10 \%$ higher than the GFDL- and NOGAPS-relative CLP5 track forecast skill levels. Relative to GFS, however, HiRAM continues to underperform through $t=$ $120 \mathrm{~h}$, with the GFS track forecast error $55 \mathrm{n}$ mi smaller than that of HiRAM at $t=120 \mathrm{~h}$. The HiRAM underperformance relative to the GFS is statistically significant at the 99th percentile for all times except $t=96$ and $120 \mathrm{~h}$.

The relative improvement in the HiRAM forecast track error between $t=72$ and $120 \mathrm{~h}$ highlights the potential of HiRAM as a useful guidance product for TC tracks at longer forecast times. Furthermore, a linear adjustment to the HiRAM forecast track based on the initial $(0 \mathrm{~h})$ forecast track error may augment HiRAM's performance with regard to track forecasting.

\section{b. Intensity}

For the 2006-09 hindcasts, HiRAM features an initial intensity error of approximately 15 knots $(\mathrm{kt}$, where $1 \mathrm{kt}=0.514 \mathrm{~m} \mathrm{~s}^{-1}$ ), which subsequently increases to $20 \mathrm{kt}$ at $t=12 \mathrm{~h}$ (Fig. 6a). The HiRAM intensity forecast error remains unchanged at $20 \mathrm{kt}$ from $t=12$ to $120 \mathrm{~h}$. When compared with its global dynamical model counterparts, HiRAM outperforms the NOGAPS and GFS intensity forecasts at all forecast times (Fig. 6a), and this outperformance is statistically significant at the 99th percentile at all forecast times (Table 2). By $t=$ $120 \mathrm{~h}$, the HiRAM forecast intensity error is more than $10 \mathrm{kt}$ smaller than either of the other two models.
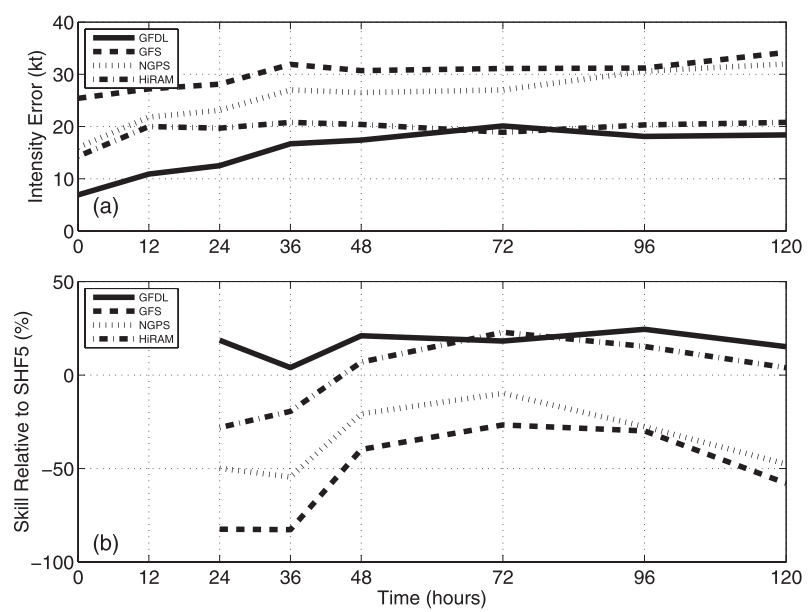

FIG. 6. (a) TC forecast intensity error (kt) and (b) TC forecast intensity skill relative to SHF5 (\%) for HiRAM, GFDL, GFS, and NOGAPS from 2006 to 2009.

A comparison of Figs. $5 b$ and $6 b$ shows that the skill of the dynamical models with regard to TC track forecasts is larger than that for TC intensity forecasts. Both the NOGAPS and GFS models feature negative skill at all forecast lead times when compared with SHF5. Between $t=24 \mathrm{~h}$ and $t=36 \mathrm{~h}$, HiRAM intensity forecasts are not skillful when compared with SHF5. Between $t=48 \mathrm{~h}$ and $t=120 \mathrm{~h}$, however, the HiRAM intensity forecast skill relative to SHF5 ranges between 5\% and 25\% (Fig. $6 \mathrm{~b})$. From $t=48$ to $120 \mathrm{~h}$, the performance of the HiRAM intensity forecast is comparable with the GFDL intensity forecast.

As seen in Fig. 7, HiRAM has a negative intensity bias that is consistent with, but smaller in magnitude than, NOGAPS and GFS over the entire forecast period. The negative HiRAM intensity bias is most likely due to the model's horizontal resolution. The current resolution is inadequate to sufficiently resolve the eyewalls of TCs, as proper simulation of fluctuations in the structure of the eyewall is necessary to produce accurate simulations of TC intensity (e.g., Knaff et al. 2007). Additionally, the vortex initialization scheme requires additional modifications in order to result in a more accurate initial intensity. An increase in horizontal resolution will most

TABLE 2. As in Table 1, but for intensity.

\begin{tabular}{ccccc}
\hline \hline Forecast time $(\mathrm{h})$ & GFDL & GFS & NOGAPS & SHF5 \\
\hline+24 & 1.00 & 1.00 & 1.00 & 0.97 \\
+36 & 0.97 & 1.00 & 1.00 & 0.90 \\
+48 & 0.85 & 1.00 & 1.00 & 0.70 \\
+72 & 0.67 & 1.00 & 1.00 & 0.95 \\
+96 & 0.66 & 1.00 & 1.00 & 0.88 \\
+120 & 0.72 & 1.00 & 1.00 & 0.58 \\
\hline
\end{tabular}




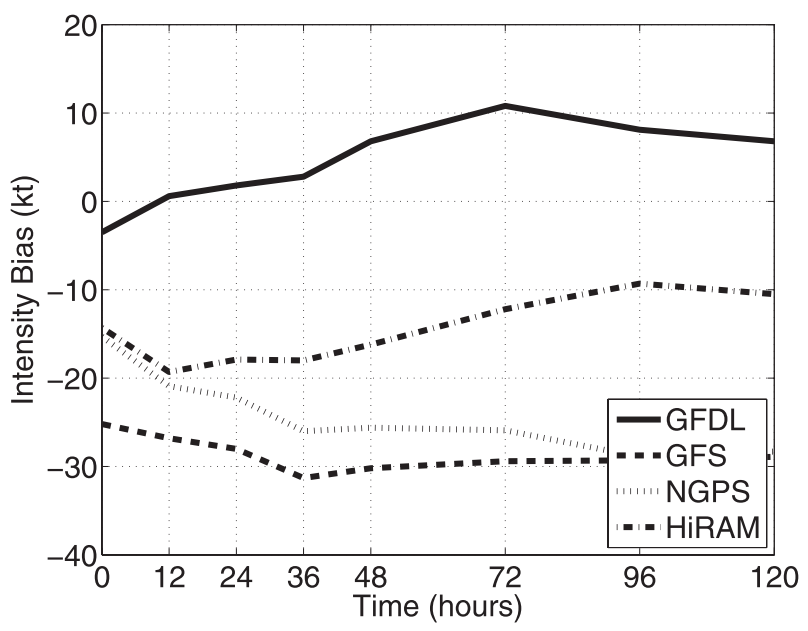

FIG. 7. TC forecast intensity bias (kt) for HiRAM, GFDL, GFS, and NOGAPS from 2006 to 2009.

likely lead to an improved initial wind-pressure relationship, which should further reduce the initial negative intensity bias.

\section{Intraseasonal forecast results}

As suggested by the results from the 5-day forecasts described in the previous section, HiRAM exhibits the largest relative forecast skill at the longest lead time in spite of its relatively crude initialization scheme. Such a result provides motivation for applying HiRAM to the forecasting of Atlantic basin TC activity on the intraseasonal time scale.

HiRAM intraseasonal forecasts are evaluated using a 21-day basin-wide forecast of total storm days (TSDs). TSDs are defined here as the number of days during which a TC has a maximum 10-m wind speed of at least tropical storm intensity (i.e., greater than $34 \mathrm{kt}$ ). TSDs are calculated using the following approach. All TC-like disturbances in the Atlantic basin are tracked in each of the HiRAM simulations using the research version of the TC tracker. Of the resulting tracks produced from the TC tracker, only track nodes featuring both a maximum wind greater than or equal to $34 \mathrm{kt}$ (i.e., tropical storm force) and a warm-core structure are included in the TSD calculation.

Rather than using results from each deterministic model forecast, a simple ensemble technique is employed, as illustrated in Fig. 8. The intraseasonal forecast is based on an average of six successive model realizations, which results in a common 21-day forecast window. The first ensemble forecast of 21-day TC activity is available at a 2-day lead time; that is, the first 21-day ensemble forecast for the period from 2 to 23 August is available on

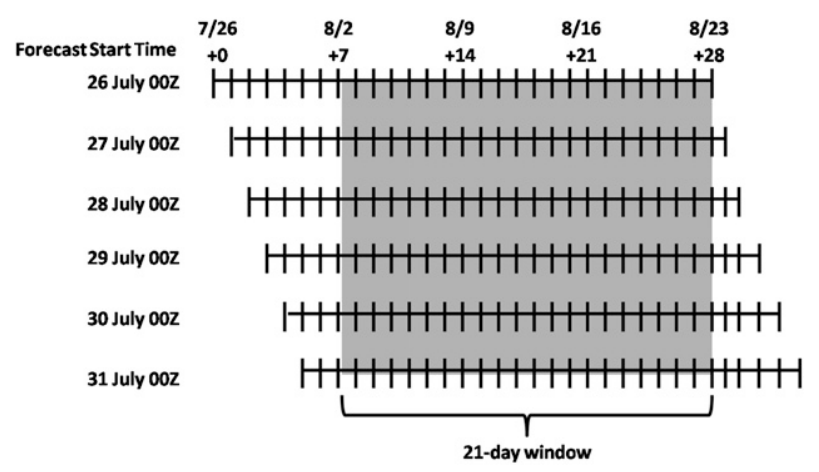

FIG. 8. An illustration of the ensemble forecasting method used in the intraseasonal HiRAM analysis. A six-member ensemble forecast for a 21-day window is available at a 2-day lead time.

31 July. The 21-day basin-wide intraseasonal forecasts are evaluated using the previously defined TSD metric. The number of cases per year (46) multiplied by four seasons (2006-2009) yields 184 total intraseasonal forecasts.

Figures $9 \mathrm{a}-\mathrm{d}$ shows time series of the daily 21-day TSDs for 2006-09. The observed TSDs for the 2007 and 2009 seasons were consistently below climatology from late July through September. For both of these seasons, HiRAM predicted below average TSDs. During the 2006 season, the TSDs for the first half of August were observed to be below average, and the HiRAM forecast agrees with the observations up to this point. The period from the end of August through the first half of September 2006 featured above average TSDs in the Atlantic. HiRAM, however, overpredicted TSDs during this period. The 2008 season featured above average activity in the Atlantic for all of August. Over most of this period, HiRAM forecasted above-average TSDs, but closer to climatology, than what is observed. Conversely, the 21-day TSD forecasts starting in early September 2008 featured below-average TSDs until the end of the forecast period. HiRAM correctly forecasted below average TSDs from 5 to 16 September, while also capturing the regime shift from above average to below average TSDs in early September.

The 2006-09 TSD forecast error was quantified using a mean square error (MSE) against the corresponding observations $\left(\mathrm{MSE}_{f}\right)$ and compared with the benchmark MSE based on climatology $\left(\mathrm{MSE}_{c}\right)$. The $\mathrm{MSE}_{f}$ and $\mathrm{MSE}_{c}$ are calculated by

$$
\begin{aligned}
& \operatorname{MSE}_{f}=\frac{1}{N} \sum_{i=1}^{N}\left[\operatorname{TSD}_{f}(i)-\operatorname{TSD}_{o}(i)\right]^{2} \text { and } \\
& \operatorname{MSE}_{c}=\frac{1}{N} \sum_{i=1}^{N}\left[\operatorname{TSD}_{c}(i)-\operatorname{TSD}_{o}(i)\right]^{2},
\end{aligned}
$$



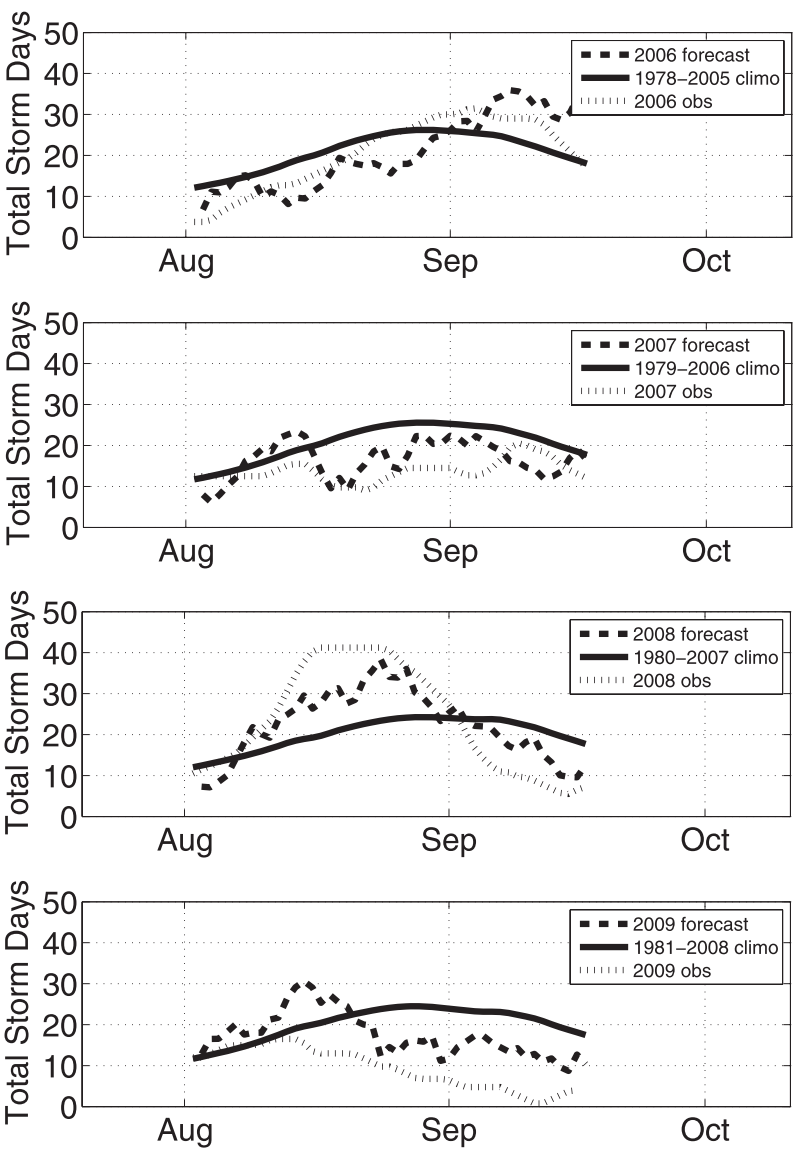

FIG. 9. Time series of the 21-day TSDs for the HiRAM forecast (thick dashed), observed (thin dashed), and preceding 28-yr climatology (solid) for the period from 2 Aug to 16 Sep (a)-(d) 2006-09.

where $N$ is the number of observations, the subscript $o$ refers to observations, and the subscript $f$ refers to forecasts. The 46 ensembles per season over four seasons results in $N=184$ observations. As seen in Table 3, $\mathrm{MSE}_{f}$ is smaller than $\mathrm{MSE}_{c}$ for all seasons except 2006. The 2006 HiRAM forecast featured the smallest $\mathrm{MSE}_{f}$ (25.7), while the HiRAM forecast with the largest $\mathrm{MSE}_{f}$ was 2009 (77.5). Given that the $\mathrm{MSE}_{c}$ for 2008 is also large, it is not surprising that the HiRAM TSD forecast is the largest for this year. Thus, the HiRAM 21-day forecasts of Atlantic basin TSDs are skillful relative to climatology over the 2006-09 seasons.

\section{Conclusions and future work}

Initial results from the HiRAM simulations at both the near-term and intraseasonal time scales appear promising. Even though the HiRAM TC forecast track error is significantly lower than the CLP5 track forecast benchmark between $t=24$ and $120 \mathrm{~h}$, it remains the
TABLE 3. Summary of the 2006-09 21-day TSD ensemble forecast MSE (MSE $)$, TSD climatological MSE $\left(\mathrm{MSE}_{c}\right)$, and the MSE difference at a 2-day lead time.

\begin{tabular}{lrrc}
\hline \hline Year & $\mathrm{MSE}_{f}$ & $\mathrm{MSE}_{c}$ & $\mathrm{MSE}_{f}-\mathrm{MSE}_{c}$ \\
\hline 2006 & 25.7 & 22.6 & 3.1 \\
2007 & 31.5 & 74.2 & -42.7 \\
2008 & 40.4 & 164.3 & -123.9 \\
2009 & 77.5 & 98.4 & -20.9 \\
All & 175.1 & 359.6 & -184.5 \\
\hline
\end{tabular}

poorest performing model with regard to forecast track error among the group of selected models up to $t=96 \mathrm{~h}$. The substantial $t=0$ track error of HiRAM demonstrates that the initialization scheme needs improvement. A decrease in the initial forecast track error will result in a decrease of forecast track error at all subsequent forecast times since initial track errors grow exponentially in time (e.g., Fraedrich and Leslie 1989; Aberson and Sampson 2003). At $t=120 \mathrm{~h}$, however, the performance of HiRAM is comparable to both NOGAPS and the GFDL hurricane model. This suggests that this early version of HiRAM will be a useful dynamical model guidance product for TC tracks at longer forecast times. Besides the relatively crude initialization procedure (as compared to other operational NWP models), the climate model heritage of HiRAM (with its emphasis on global hydrological and energy balance, cloud-radiation interaction, and no specific tuning for weather prediction) may also contribute to improved relative skill at longer lead times.

With regard to the near-term HiRAM intensity forecast error, HiRAM outperformed the SHF5 forecast intensity benchmark between $t=48$ and $120 \mathrm{~h}$. The underperformance up to $t=48 \mathrm{~h}$ is related to initialization issues. HiRAM intensity forecasts consistently outperformed NOGAPS and GFS intensity forecasts at all lead times. Besides improved numerics and physical parameterizations, much of this outperformance may be attributed to the finer horizontal resolution of HiRAM relative to NOGAPS $(55 \mathrm{~km})$ and GFS (30 km). It should also be noted that the HIRAM intensity forecast error is similar to the GFDL's hurricane model between 72 and $120 \mathrm{~h}$, which highlights the utility of HiRAM as an intensity guidance product at longer forecast lead times. While the HiRAM track and intensity forecast results are promising, additional cases are needed to further assess statistical significance.

The 21-day ensemble forecasts of basin-wide TC activity in the Atlantic at a 2-day lead time are demonstrated to be skillful relative to the climatological forecasts for the same period. The forecast MSE is smaller than the climatological MSE for the entire 2006-09 period, and for each individual year except 2006. 
Results obtained from the 2006-09 hindcasts provide a baseline with which to compare future HiRAM upgrades. The following modifications to HiRAM will be implemented over the next year, and are discussed below.

- An improved initialization technique making use of an ensemble Kalman filter (EnKF) approach

An advanced data assimilation system based on the EnKF methodology is currently being developed in conjunction with the NOAA Hurricane Forecast Improvement Project (HFIP). This system is particularly advantageous for ensemble forecasts at intraseasonal (or longer) time scales. The EnKF approach can be combined with an improved vortex breeding scheme to provide better initial conditions for nearterm TC forecasts. Whitaker et al. (2006) implemented an EnKF system using the GFS model, and demonstrated that the ensemble data assimilation system outperformed the operational three-dimensional variational data assimilation (3DVAR) GFS initialization system, especially in data-sparse regions. Given the data sparsity in the tropics, the implementation of an EnKF initialization scheme in HiRAM should lead to a reduction in initial errors of Atlantic basin near-term TC track and intensity forecasts.

Further, during the initial development of HiRAM, analysis of the 500-mb anomaly correlation coefficient was performed. The HiRAM forecast score was acceptable, but underperformed global forecasting models with a data assimilation system (e.g., ECMWF). It is believed that when the data assimilation system described above is in place for HiRAM, the already acceptable anomaly correlation coefficient will improve significantly.

- Increased horizontal resolution

Although the 25-km version of HiRAM used in this study is shown to provide skillful intensity forecasts, the horizontal resolution remains inadequate for properly resolving typical TCs (e.g., Hill and Lackmann 2009; Gentry and Lackmann 2010). Further, the vortex breeding scheme may require a finer horizontal resolution to initialize intense hurricanes. Preliminary simulations of several category 5 (CAT5) hurricane cases using 13-km resolution (results not shown) demonstrate significant improvements in track and intensity forecasts relative to identical simulations run at a horizontal resolution of $25 \mathrm{~km}$. HiRAM will be upgraded to at least a horizontal resolution of $13 \mathrm{~km}$ in future studies provided that a sufficient amount of computer resources are available.

\section{- Coupling to a 3D ocean model}

It is well known that coupling a high-resolution atmospheric model to a $3 \mathrm{D}$ ocean model leads to substantial improvements in the prediction of TC intensity when compared with a high-resolution atmospheric model run with prescribed SSTs (e.g., Bender et al. 1993; Bender and Ginis 2000). As the resolution increases to a level that can support compact and strong TCs, it then becomes imperative that atmosphere-ocean interaction be accounted for in the forecast model, and to a lesser degree, in the assimilation system. Leaving a high-resolution atmospheric model detached from the ocean often leads to the overprediction of TC intensity.

\section{- Coupling to a wave model}

Given HiRAM's origins as a climate model, the current surface drag parameterization in HiRAM was not tuned for high surface wind conditions. Thus, coupling HiRAM to a wave model should improve the surface drag formulation at higher wind speeds (e.g., Moon et al. 2004). Additionally, coupling to a wave model will also improve TC intensity forecasts owing to a more realistic representation of the air-sea interface (e.g., Fan et al. 2009).

The HiRAM formulation presented in this paper provides a baseline with which to compare all future modifications. Results from HiRAM hindcasts at different temporal scales (from short term to seasonal) incorporating the aforementioned modifications will be discussed in subsequent papers and compared to the baseline results herein.

Acknowledgments. We thank Ming Zhao and Isaac Held for helpful discussions and guidance during the course of this study. This research was supported by a grant from WeatherPredict Consulting, Inc., an affiliate of RenaissanceRe. Additionally, Isaac Ginis, S.-J. Lin, and Timothy Marchok thank NOAA's Hurricane Forecast Improvement Project for additional support.

\section{REFERENCES}

Aberson, S. D., 1998: Five-day tropical cyclone track forecasts in the North Atlantic basin. Wea. Forecasting, 13, 1005-1015.

— , and C. R. Sampson, 2003: On the predictability of tropical cyclone tracks in the northwest Pacific basin. Mon. Wea. Rev., 131, 1491-1497.

Anderson, J., and Coauthors, 2004: The new GFDL global atmosphere and land model AM2-LM2: Evaluation with prescribed SST simulations. J. Climate, 17, 4641-4673.

Barnes, S. L., 1964: A technique for maximizing details in numerical weather map analysis. J. Appl. Meteor., 3, 396-409. 
Belanger, J. I., P. J. Webster, and J. A. Curry, 2010: Predictability of North Atlantic tropical cyclone activity on intraseasonal time scales. Mon. Wea. Rev., 138, 4362-4374.

Bender, M. A., and I. Ginis, 2000: Real-case simulations of hurricaneocean interaction using a high-resolution coupled model: Effects on hurricane intensity. Mon. Wea. Rev., 128, 917-946.

,$- \ldots$, and Y. Kurihara, 1993: Numerical simulations of tropical cyclone-ocean interaction with a high-resolution coupled model. J. Geophys. Res., 98 (D12), 23 245-23 263.

— - _ R. Tuleya, B. Thomas, and T. Marchok, 2007: The operational GFDL coupled hurricane-ocean prediction system and summary of its performance. Mon. Wea. Rev., 135, 3965-3989.

Camargo, S. J., A. G. Barnston, P. J. Klotzbach, and C. W. Landsea, 2007: Seasonal tropical cyclone forecasts. WMO Bull., 56, 297-309.

Côté, J., S. Gravel, A. Mthot, A. Patoine, M. Roch, and A. Staniforth, 1998: The operational CMC-MRB Global Environmental Multiscale (GEM) model. Part I: Design considerations and formulation. Mon. Wea. Rev., 126, 1373-1395.

Cullen, M. J. P., 1993: The Unified Forecast/Climate Model. Meteor. Mag., 122, 81-122.

Davis, C., and Coauthors, 2008: Prediction of landfalling hurricanes with the Advanced Hurricane WRF model. Mon. Wea. Rev., 136, 1990-2005.

DeMaria, M., J. A. Knaff, and C. R. Sampson, 2007: Evaluation of long-term trend in tropical cyclone intensity forecasts. Meteor. Atmos. Phys., 97, 19-28.

Elsberry, R. L., M. S. Jordan, and F. Vitart, 2010: Predictability of tropical cyclone events on intraseasonal timescales with the ECMWF monthly forecast model. Asia-Pac. J. Atmos. Sci., 10, $1-10$.

Fan, Y., I. Ginis, and T. Hara, 2009: The effect of wind-wavecurrent interaction on air-sea momentum fluxes and ocean response in tropical cyclones. J. Phys. Oceanogr., 39, 1019-1034.

Fraedrich, K., and L. M. Leslie, 1989: Estimates of cyclone track predictability. I: Tropical cyclones in the Australian region. Quart. J. Roy. Meteor. Soc., 115, 79-92.

Franklin, J., 2010: 2009 National Hurricane Center forecast verification report. NOAA/NWS/NCEP/National Hurricane Center/ Tropical Prediction Center, 71 pp.

Gentry, M. S., and G. M. Lackmann, 2010: Sensitivity of simulated tropical cyclone structure and intensity to horizontal resolution. Mon. Wea. Rev., 138, 688-704.

Goerss, J. S., and R. A. Jeffries, 1994: Assimilation of synthetic tropical cyclone observations into the Navy Operational Global Atmospheric Prediction System. Wea. Forecasting, 9, 557-576.

Heming, J. T., J. C. L. Chan, and A. M. Radford, 1995: A new scheme for the initialization of tropical cyclones in the U.K. Meteorological Office Global Model. Meteor. Appl., 2, 171-184.

Hill, K. A., and G. M. Lackmann, 2009: Analysis of idealized tropical cyclone simulations using the Weather Research and Forecasting model: Sensitivity to turbulence parameterization and grid spacing. Mon. Wea. Rev., 137, 745-765.

Hogan, T. F., and T. E. Rosmond, 1991: The description of the Navy Operational Global Atmospheric Prediction System's Spectral Forecast Model. Mon. Wea. Rev., 119, 1786-1815.

Klotzbach, P. J., 2010: On the Madden-Julian oscillation-Atlantic hurricane relationship. J. Climate, 23, 282-293.

Knaff, J. A., M. DeMaria, C. R. Sampson, and J. M. Gross, 2003: Statistical, 5-day tropical cyclone intensity forecasts derived from climatology and persistence. Wea. Forecasting, 18, 204-223.

, C. R. Sampson, M. DeMaria, T. P. Marchok, J. M. Gross, and C. J. McAdie, 2007: Statistical tropical cyclone wind radii prediction using climatology and persistence. Wea. Forecasting, 22, 781-791.

LeRoy, A., and M. C. Wheeler, 2008: Statistical prediction of weekly tropical cyclone activity in the Southern Hemisphere. Mon. Wea. Rev., 136, 3637-3654.

Lin, S.-J., 2004: A "vertically Lagrangian" finite-volume dynamical core for global models. Mon. Wea. Rev., 132, 2293-2307.

Lin, X., and Coauthors, 2006: A view of Hurricane Katrina with early 21 st century technology. Eos, Trans. Amer. Geophys. Union, 87, doi:10.1029/2006EO410002.

Lin, Y. L., R. D. Farley, and H. D. Orville, 1983: Bulk parameterization of the snow field in a cloud model. J. Appl. Meteor., 22, 1065-1092.

Lord, S. J., 1993: Recent developments in tropical cyclone track forecasting with the NMC global analysis and forecast system. Preprints, 20th Conf. on Hurricanes and Tropical Meteorology, San Antonio, TX, Amer. Meteor. Soc., 290-291.

Marchok, T. P., 2002: How the NCEP tropical cyclone tracker works. Preprints, 25th Conf. on Hurricanes and Tropical Meteorology, San Diego, CA, Amer. Meteor. Soc., P1.13. [Available online at http://ams.confex.com/ams/25HURR/techprogram/ paper_37628.htm.]

Miura, H., M. Satoh, T. Nasuno, A. T. Noda, and K. Oouchi, 2007: A Madden-Julian oscillation event realistically simulated by a global cloud-resolving model. Science, 318, 1763-1765.

Moon, I.-J., I. Ginis, and T. Hara, 2004: Effect of surface waves of air-sea momentum exchange. Part II: Behavior of drag coefficient under tropical cyclones. J. Atmos. Sci., 61, 2334-2348.

Putman, W. M., and S. J. Lin, 2007: Finite-volume transport on various cubed-sphere grids. J. Comput. Phys., 227, 55-78.

Roundy, P. E., 2008: Probabilistic forecasting of tropical cyclones utilizing wave and climate modes. Preprints, 28th Conf. on Hurricanes and Tropical Meteorology, Orlando, FL, Amer. Meteor. Soc., 10B.7. [Available online at http://ams.confex. com/ams/28Hurricanes/techprogram/paper_137364.htm.]

_ and C. J. Schreck, 2009: A combined wavenumber-frequency and time extended EOF approach for tracking the progress of modes of large-scale organized convection. Quart. J. Roy. Meteor. Soc., 135, 161-173.

Shen, B.-W., R. Atlas, O. Reale, S.-J. Lin, J. D. Chern, J. Chang, and C. Henze, 2006: Hurricane forecasts with a global mesoscale-resolving model: Preliminary results with Hurricane Katrina (2005). Geophys. Res. Lett., 33, L13813, doi:10.1029/ 2006GL026143.

Vitart, F., 2004: Monthly forecasting at ECMWF. Mon. Wea. Rev., 132, 2761-2779.

—, J. L. Anderson, and W. F. Stern, 1997: Simulation of interannual variability of tropical storm frequency in an ensemble of GCM integrations. J. Climate, 10, 745-760.

— A. Leroy, and M. C. Wheeler, 2010: A comparison of dynamical and statistical predictions of weekly tropical cyclone activity in the Southern Hemisphere. Mon. Wea. Rev., 138, 3671-3682.

Whitaker, J. S., X. Wei, and F. Vitart, 2006: Improving week-2 forecasts with multimodel reforecast ensembles. Mon. Wea. Rev., 134, 2279-2284.

Wilks, D. S., 2006: Statistical Methods in the Atmospheric Sciences. International Geophysics Series, Vol. 59, Academic Press, 627 pp.

Zhang, C., 2005: Madden-Julian oscillation. Rev. Geophys., 43, 1-36.

Zhao, M., I. M. Held, S.-J. Lin, and G. A. Vecchi, 2009: Simulations of global hurricane climatology, interannual variability, and response to global warming using a $50-\mathrm{km}$ resolution GCM. J. Climate, 22, 6653-6678. 